\title{
The ABCDEF Bundle for the Respiratory Therapist
}

\author{
Matthew F Mart, Nathan E Brummel, and E Wesley Ely
}

\author{
Introduction \\ Assess, Prevent, and Manage Pain \\ Both Spontaneous Awakening Trials and Spontaneous Breathing Trials \\ Choice of Analgesia and Sedation \\ Delirium: Assessment, Prevention, and Management \\ Early Mobility and Exercise \\ Family Engagement and Empowerment \\ The ABCDEF Bundle: Synergistic Care Improving Outcomes \\ Conclusions and Future Directions
}

\begin{abstract}
The clinical approach to the critically ill patient has changed dramatically over the last several decades from one of deep sedation to that of mobilizing patients on mechanical ventilation and limiting sedation. The ABCDEF bundle is a multidisciplinary, evidence-based approach to the holistic management of critically ill patients that aims to optimize patient recovery, minimize iatrogenesis, and engage and empower the patient and family during their hospitalization. To achieve this goal, the bundle includes assessments for pain, delirium, and readiness to stop sedation and to start spontaneous breathing trials. It also encourages early mobilization of the patient, avoidance of restraints, and engagement with the family in bedside rounds to improve communication. Performance of this bundle reduces mortality, ventilator days, intensive care readmissions, delirium, coma, restraint use, and discharge to facilities in a dose-dependent manner. The respiratory therapist, as a key member of the critical care team, is essential to the implementation, performance, and success of the ABCDEF bundle. This review aims to describe each component of the ABCDEF bundle, provide evidence for both the impact of individual interventions as well as the entire bundle, and detail the importance of this multidisciplinary approach to the care of the critically ill patient. Key words: abcdef bundle; analgesia; sedation; critical care; delirium; early mobility; family engagement; respiratory therapy; intensive care unit; intensive care unit liberation; mechanical ventilation. [Respir Care 2019;64(12):1561-1573. (c) 2019 Daedalus Enterprises]
\end{abstract}

\section{Introduction}

Millions of adults are admitted to ICUs in the United States each year, ${ }^{1}$ with improving survival rates despite

Drs Mart, Brummel, and Ely are affiliated with the Critical Illness, Brain Dysfunction, and Survivorship (CIBS) Center, and the Division of Allergy, Pulmonary, and Critical Care Medicine, Department of Medicine, Vanderbilt University Medical Center, Nashville, Tennessee. Dr Brummel is affiliated with the Division of Pulmonary, Critical Care, and Sleep Medicine, The Ohio State University Wexner Medical Center, Columbus, Ohio. Tennessee. Dr Ely is also affiliated with the Center for Health increasing severity of illness. ${ }^{2}$ With falling mortality, critical care medicine now faces another growing challenge in the long-term cognitive, physical, and psychiatric impairments that follow critical illness. ${ }^{3-6}$ Given the growing recognition of these impairments and their link to in-hospital complications such as delirium, the

Services Research, The Institute for Medicine and Public Health, Vanderbilt University Medical Center, and the Tennessee Valley Veterans Affairs Healthcare System, Geriatric Research Education and Clinical Centers (GRECC), Nashville, Tennessee. 


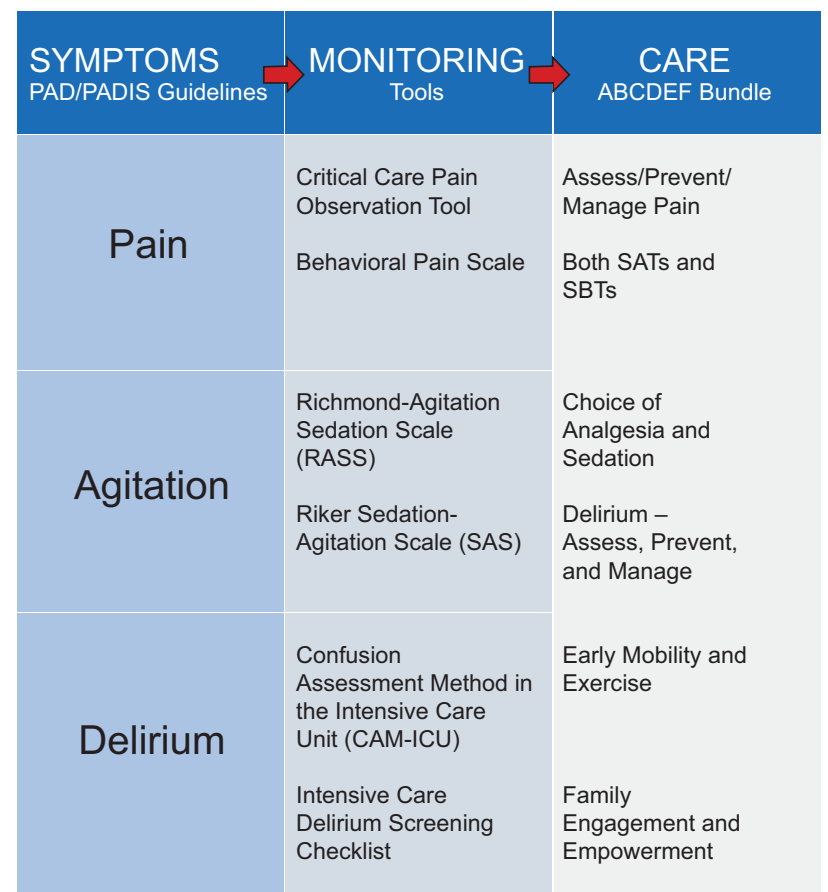

Fig. 1. ICU liberation: pain, agitation, and delirium guidelines and the concept of the ABCDEF bundle. From Reference 2, with permission.

first clinical practice guidelines for the management of pain, agitation, and delirium in adults patients in the intensive care unit (ICU PAD) were issued. ${ }^{7}$ These guidelines provided a framework for managing pain, agitation, and delirium in concordance with the most recent evidence highlighting the impact of sedation practices and delirium on the outcomes of ICU survivors. These recommendations were updated in the most recent 2018 Prevention and Management of Pain, Agitation/Sedation, Delirium, Immobility, and Sleep Disruption in the Adult Patients in the ICU (PADIS) Guidelines. ${ }^{8}$ These same evidence-based concepts were built into the $\mathrm{ABCDE}$, and now ABCDEF, bundle as part of the ICU liberation campaign from the Society of Critical Care Medicine (SCCM) (Fig. 1). ${ }^{2,9,10}$ The ABCDEF bundle serves as a guide for multidisciplinary critical care, in-

This work was supported by the National Institutes of Health (grants T32HL087738, 1R01AG058639, and 1K76AG054864), and the Department of Veterans Affairs Tennessee Valley Healthcare System Geriatric Research, Education and Clinical Center.

Correspondence: Matthew F Mart MD, Division of Allergy, Pulmonary, and Critical Care Medicine, Department of Medicine, Vanderbilt University Medical Center, Nashville, TN 37212. E-mail: matthew.f.mart@vumc.org.

DOI: $10.4187 /$ respcare. 07235
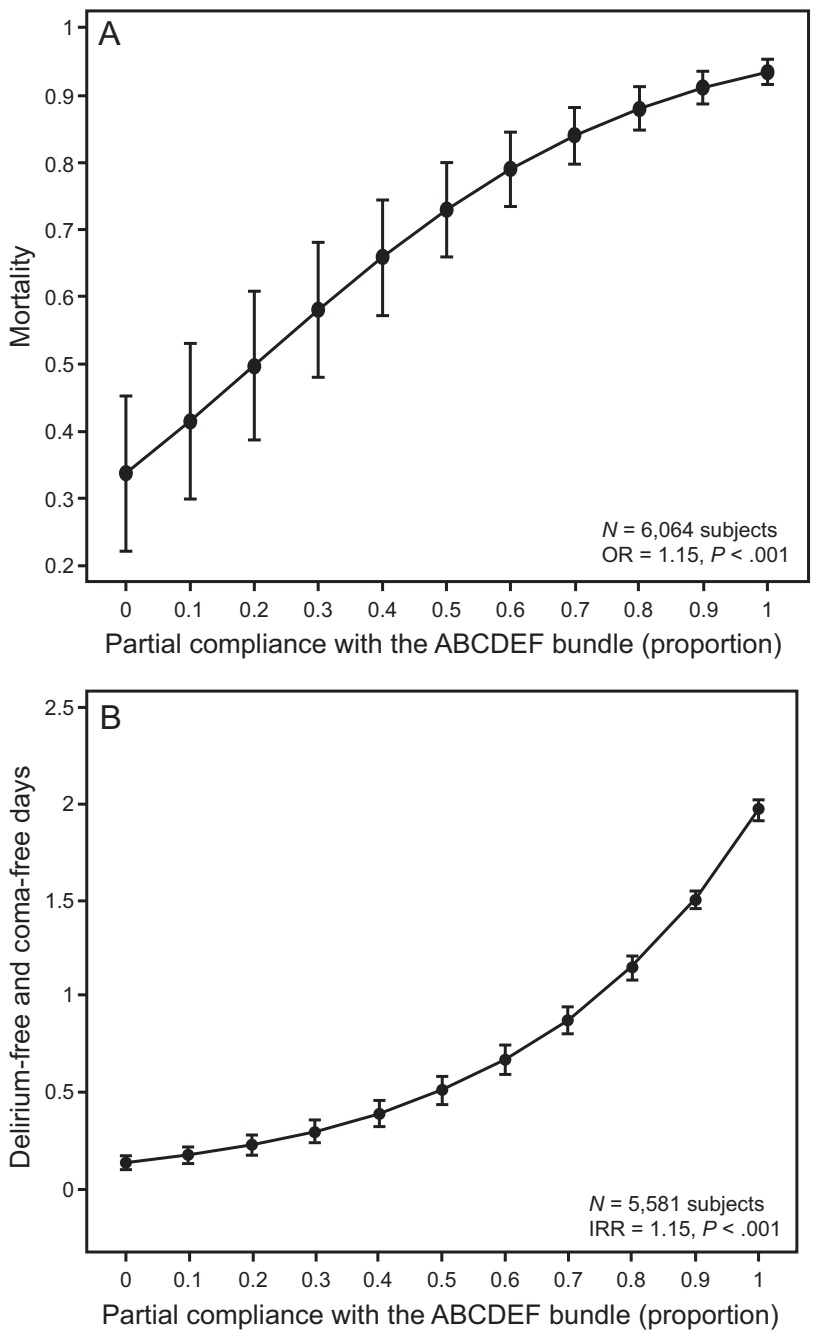

Fig. 2. A: ABCDEF bundle compliance and mortality. B: deliriumfree and coma-free days. OR = odds ratio; IRR = incidence rate ratio. From Reference 80, with permission.

corporating diverse specialties, including respiratory therapists (RTs), as partners in improving care for the sickest patients. The key components of the ABCDEF bundle are: (1) Assess, prevent, and manage pain; (2) $B$ oth spontaneous awakening trials (SAT) and spontaneous breathing trials (SBT); (3) Choice of analgesia and sedation; (4) Delirium - Assessment, Prevention, and Management; (5) Early Mobility and Exercise; and (6) Family engagement and empowerment. ${ }^{2}$ The ABCDEF bundle has been demonstrated to improve outcomes in multiples studies (Fig. 2). Compliance with the bundle is associated increased survival, reduced delirium, fewer days on mechanical ventilation, and fewer ICU readmissions in a dose-dependent manner. ${ }^{11}$ This review will discuss the evidence and implementation of each portion of the bundle, highlighting the benefits and remaining questions regarding the bundle and emphasizing the multidisciplinary nature of its success. 


\section{Assess, Prevent, and Manage Pain}

Pain is a common, often daily, occurrence that can go unrecognized and undertreated in all types of ICUs. ${ }^{12}$ For example, prior to performance of various ICU procedures, pain is assessed only $35 \%$ of the time, ${ }^{13}$ although most ICU procedures cause a significant increase in pain. ${ }^{14}$ Pain is also a significant risk factor for the development of delirium in the critically ill. ${ }^{15-17}$ It is essential for the critical care team to actively assess and manage pain in the ICU to prevent complications and reduce suffering.

Critically ill patients are frequently sedated due to medication or their disease process and often are on mechanical ventilation, limiting their ability to report pain reliably. Due to those limitations, other methods of assessing pain are necessary in the ICU. There are two widely accepted and validated pain scales that utilize behavior and physiological measures to assess pain: the Behavioral Pain Scale, ${ }^{17}$ and the Critical-Care Pain Observation Tool. ${ }^{18,19}$ Both pain scales utilize a variety of bedside indicators to assess pain, such as facial expressions, body movements, and synchrony with the ventilator. A Behavioral Pain Scale score $\geq 5$ or Critical-Care Pain Observation Tool score $\geq 3$ represent significant pain that should be treated. The Critical-Care Pain Observation Tool has been shown to be feasible and simple to use at the bedside, ${ }^{20}$ and it improved the routine assessment of pain by nursing staff. ${ }^{21}$ Although these assessments are most often performed by nursing staff, RTs are critical in identifying manifestations of pain, which can include grimacing, asynchrony with the ventilator, and agitation.

When significant pain is identified by the care team, the preferred first-line pain management is the use of intravenous opioids. ${ }^{8}$ Adjunctive methods of pain control, such as nonsteroidal anti-inflammatory drugs and gabapentin, should also be considered and used in patients to reduce opioid doses in addition to nonpharmacologic measures that can reduce pain. ${ }^{22}$ Additionally, preprocedural analgesia should be provided to patients undergoing painful procedures or testing to limit discomfort. Adequately managing pain reduces delirium and facilitates performance of other components of the ABCDEF bundle, such as early mobilization and breathing trials.

\section{Both SATs and SBTs}

Coordinated and protocolized SATs and SBTs are foundational to liberation from mechanical ventilation and comprise an important part of the ABCDEF bundle. SATs involve the cessation of sedatives and narcotics in patients who pass an initial safety screen. Kress and colleagues ${ }^{23}$ demonstrated in a single-center, randomized, controlled trial of 128 subjects receiving mechanical ventilation that daily interruption of sedation and narcotics compared to usual care led to a significant decrease in ventilator days and improved ICU length of stay. In the same cohort, subjects in the intervention group had fewer symptoms related to post-traumatic stress disorder upon long-term follow-up than the usual care group. ${ }^{24}$ In a secondary analysis, subjects in the SAT group also had fewer instances of ventilator-associated pneumonia and other complications of ICU care. ${ }^{25}$ Similarly, deep sedation during critical illness has been associated with higher long- and short-term mortality and prolonged mechanical ventilation independent of severity of illness. ${ }^{26,27}$ Routine use of SATs reduces the overall dose of sedatives and narcotics and reduces time to liberation from mechanical ventilation and mortality.

Similar to the use of SATs, the routine use of SBTs facilitates ventilator weaning and liberation. Esteban et $\mathrm{al}^{28}$ reported that the use of breathing trials (2-h T-piece trials vs pressure support trials) reduced time on mechanical ventilation and was an acceptable method of discontinuing ventilator support. Subsequent work by Ely and colleagues ${ }^{29}$ demonstrated that daily screening and performance of SBTs resulted in a median of $4.5 \mathrm{~d}$ of mechanical ventilation compared to $6 \mathrm{~d}$ of mechanical ventilation in the control arm. In the multi-center, randomized, controlled Awakening and Breathing Controlled (ABC) trial, subjects randomized to a protocol of paired daily SATs and SBTs had fewer ventilator days $(14.7 \mathrm{~d}$ vs $11.6 \mathrm{~d}$; mean difference: $3.1 \mathrm{~d}, 95 \%$ CI 0.7-5.6) and shorter ICU and hospital length of stay compared to subjects randomized to a standard SBT protocol. This benefit extended beyond the acute hospitalization: subjects in the paired SAT/SBT group had lower mortality in the year following critical illness with a number needed to treat of 7.4 (hazard ratio $0.68,95 \% \mathrm{CI}$ $0.50-0.192, P=.1) .^{30}$

The use of protocolized paired SAT and SBT trials involves a coordinated set of steps to assess the safety of performing both the SAT and SBT individually (Fig. 3). If the patient passes the safety screen for the SAT, the SAT is then performed by the bedside critical care nurse by stopping any parenteral sedatives and narcotics. The bedside nurse subsequently assesses for signs of SAT failure as described in Figure 3. If the patient fails the SAT, sedation and analgesia is restarted at half the original dose and titrated to a specified goal. If the patient passes criteria for the SAT, the respiratory therapist will then perform an SBT safety screen. Similar to the screening process for the SAT, should the patient fail any of the safety criteria, then the patient remains on full ventilatory support and the steps are repeated again the next day. If the patient passes the safety screen, the SBT is attempted. The use of pressure support ventilation versus T-piece trials are often institution-dependent. Esteban et $\mathrm{al}^{28}$ showed that the rate of successful extubation (ie, remaining extubated for $48 \mathrm{~h}$ after an SBT) did not differ between the T-piece group and the pressure support ventilation group. Once the patient is on an SBT, the respiratory therapist then assesses the pa- 


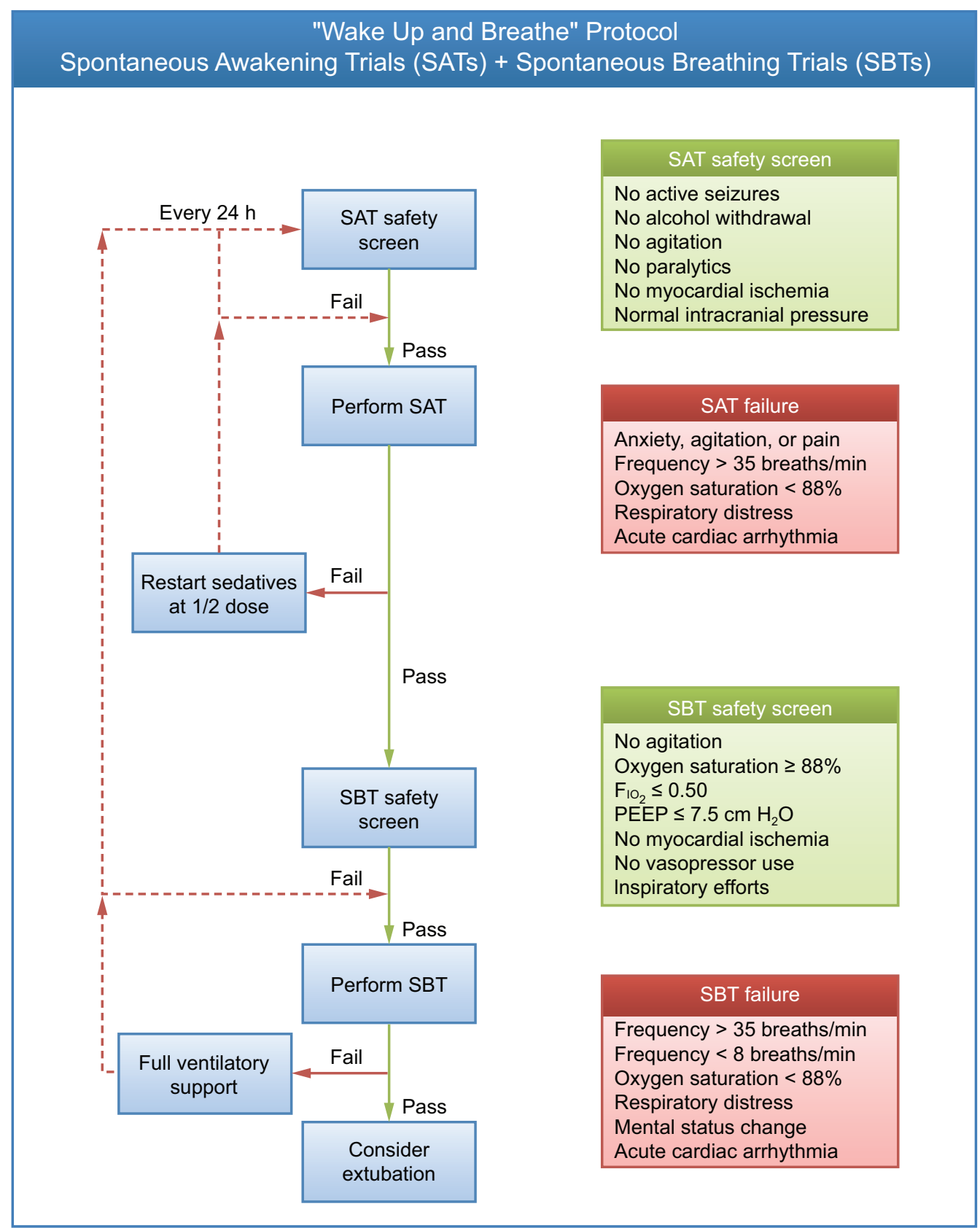

Fig. 3. Wake up and breathe protocol: paired spontaneous awakening trials (SATs) and spontaneous breathing trials (SBTs). Courtesy Vanderbilt University Medical Center and icudelirium.org.

tient for any signs of SBT failure. If the patient shows any signs of SBT failure, the patient is then switched back to full mechanical ventilatory support. If the patient shows no sign of failure and tolerates $30-120 \mathrm{~min}$ of the SBT, the RT then informs the medical team and bedside nurse that the patient meets extubation criteria. The optimum duration of SBT is debated, although studies suggest that $30 \mathrm{~min}$ is likely sufficient and not different than 120-min trials. ${ }^{31}$ Once notified that the patient meets extubation criteria, the critical care physician at that time should consider extubation. Ultimately, the coordinated, multidisciplinary performance of SATs and SBTs as a part of the ABCDEF bundle results in earlier successful removal of mechanical ventilation and reduces ICU morbidity. RTs play an important role in helping liberate patients from ventilatory support.

\section{Choice of Analgesia and Sedation}

The $C$ in the ABCDEF bundle refers to the choice of analgesia and sedation, consistent with the most recent 


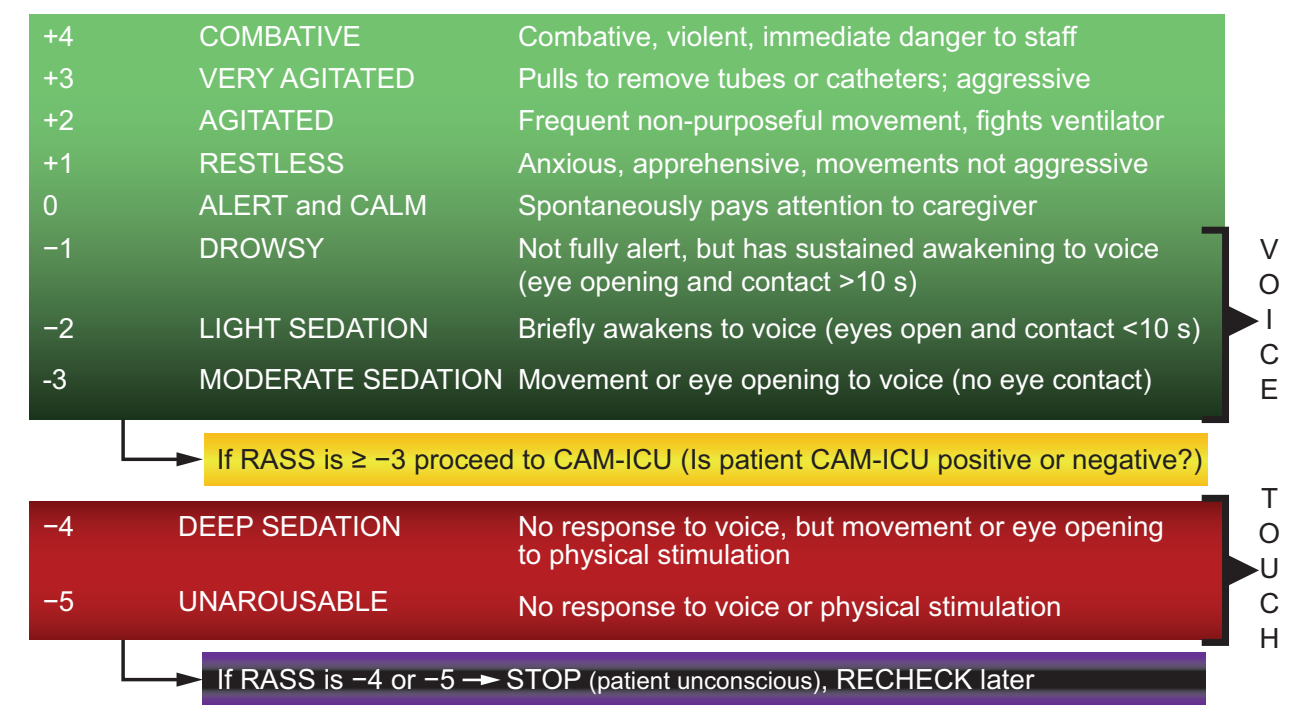

Fig. 4. Richmond Agitation-Sedation Scale (RASS). CAM-ICU = confusion assessment method for the ICU. From Reference 33, with permission.

PADIS guidelines. The focus is on goal-directed sedation and analgesia that reduces the overall drug burden and achieves light sedation. This includes routine assessment of pain in all ICU patients, utilizing validated measures as noted above as well as routine monitoring of level of consciousness and sedation depth. The recommended sedation scales from the PADIS guidelines include the Richmond Agitation-Sedation Scale (RASS) (Fig. 4) and the Riker Sedation-Agitation Scale (SAS). These instruments are similar in using graded point scales to describe patients level of consciousness from comatose (SAS $=1$ or RASS $=-5$ ) to extreme agitation (SAS $=7$ or RASS $=+4$ ). ${ }^{32}$ The RASS, for example, has exhibited reliability across multiple ICU settings and disciplines. ${ }^{33}$ The goal of analgesia and sedation in the critically ill patient should be a calm and alert state, allowing for patient-clinician and patientfamily interactions but also facilitating ongoing medical support as needed in the ICU. Targeting light sedation (ie, a calm and alert level of consciousness) demonstrates a trend toward a shorter time to extubation and a reduced need for tracheostomy, ${ }^{8,34-36}$ and therefore should be the standard sedation goal in the absence of other extenuating clinical concerns necessitating deep sedation.

Pain control is considered the first-line therapy prior to considering the addition of sedating agents, which is a concept known as analgosedation (ie, an analgesia-first approach while limiting sedation as clinically appropriate). Parental opioids are the preferred first-line medications for pain control, and often the desired sedation depth, as well as pain control, can be achieved with analgesia alone. ${ }^{8}$ Should further sedation be needed for either patient comfort or for clinical indication, the guidelines recommend avoidance of benzodiazepines in favor of other agents such as dexmedetomidine or propofol and limitation of overall sedation exposure to the greatest extent possible. Higher exposure to sedatives is linked to greater mortality, longer time on mechanical ventilation, and delirium. ${ }^{26,37-39}$ The use of benzodiazepines has been reported to be an independent risk factor for the development of delirium in the ICU. ${ }^{40,41}$ Compared with the use of dexmedetomidine, the use of benzodiazepines was associated in multiple studies with fewer delirium-free days. ${ }^{42,43}$

While the PADIS Guidelines suggest the use of nonbenzodiazepine sedatives, they provide limited guidance on a specific non-benzodiazepine agent of choice due to a research gap in the benefits and limitations of individual sedation agents. Based on several studies suggesting that propofol reduces time to extubation compared to benzodiazepines, ${ }^{4-46}$ the PADIS guidelines make a conditional recommendation for the use of propofol over benzodiazepines in critically ill patients who require sedation after cardiac surgery. In non-cardiac surgery patients undergoing mechanical ventilation, the parallel MIDEX-PRODEX trials evaluated whether dexmedetomidine was non-inferior to midazolam and propofol, respectively, in terms of time to target sedation and median duration of mechanical ventilation. Dexmedetomidine was found to be non-inferior to both midazolam and propofol in terms of time to target sedation without rescue medication and was associated with decreased duration of mechanical ventilation compared to midazolam but not propofol. ${ }^{47}$ The PADIS guidelines suggest the use of either dexmedetomidine or propofol over benzodiazepines in adults receiving mechanical ventilation, noting a low quality of evidence and the remaining unanswered questions regarding sedation choice. ${ }^{8}$ Ultimately, the choice of analgesia and sedation is focused on achieving light sedation through analgosedation. 


\section{Confusion Assessment Method for the ICU (CAM-ICU) Flowsheet}

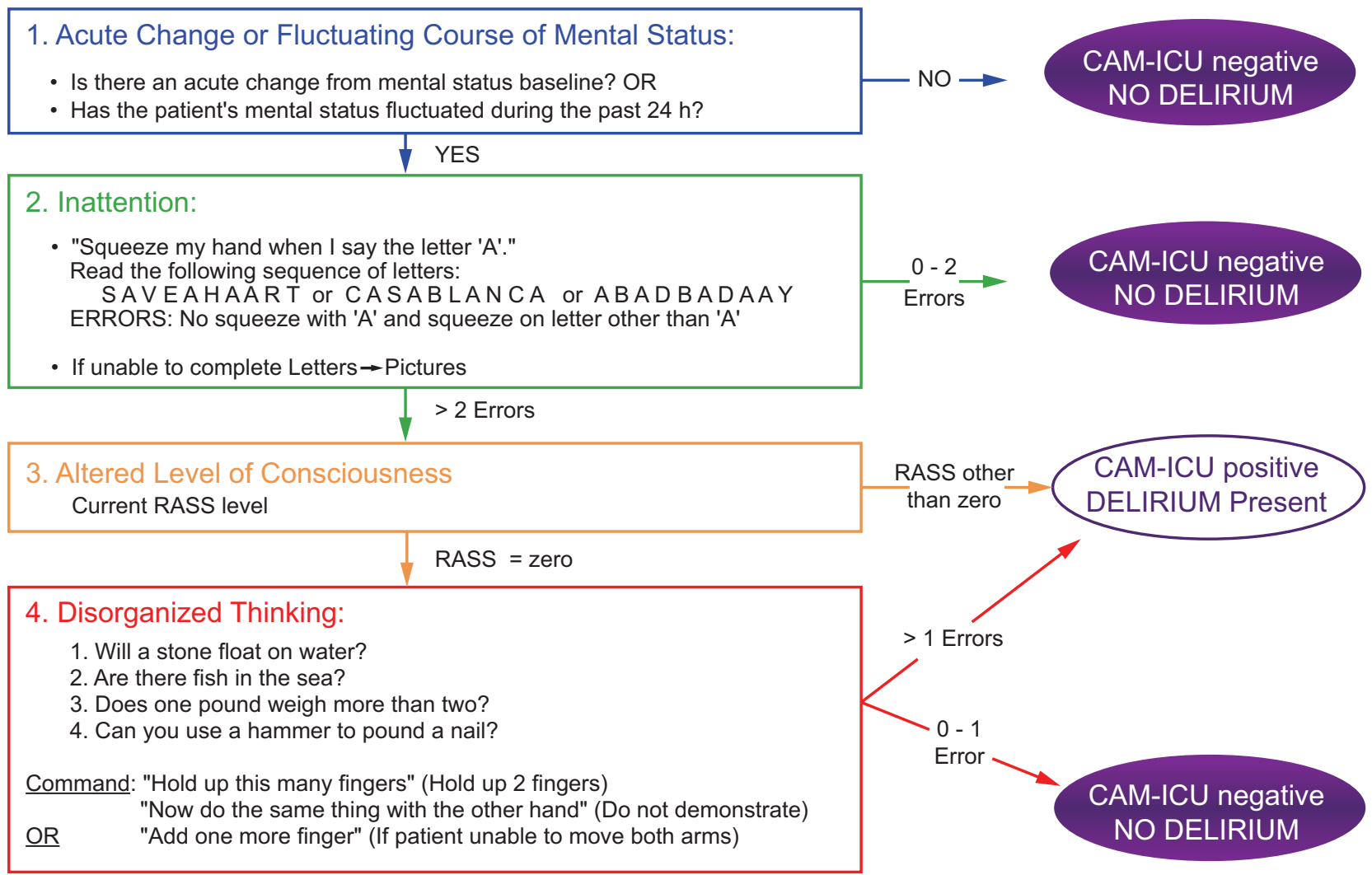

Fig. 5. Confusion assessment method for the ICU (CAM-ICU). RASS = Richmond Agitation-Sedation Scale. Adapted from Reference 102, with permission. Courtesy Dr Ely and Vanderbilt University.

\section{Delirium: Assessment, Prevention, and Management}

Delirium, the $D$ in the ABCDEF bundle, is a pervasive form of acute brain failure in the critically ill and an important area of clinical investigation and management. Delirium is defined as an acute change in awareness and in attention that develops within hours to days with a waxing and waning course that is not explained by a preexisting neurocognitive disorder. ${ }^{48}$ Patients can develop hypoactive delirium, defined by a reduced level of consciousness with fluctuating attention and awareness, or hyperactive delirium, defined by increasing levels of agitation with fluctuating attention and awareness. Patients can also develop mixed delirium, consisting of both hyper- and hypoactive forms. ${ }^{49}$ Delirium is associated with increased mortality and long-term complications, such as long-term cognitive impairment, which is an acquired dementia after critical illness. ${ }^{3,50-52}$ The etiology of delirium is multifactorial, and certain precipitating factors include the use of sedating medications (particularly benzodiazepines), hypoxemia, sepsis, untreated pain, prolonged immobiliza- tion, sleep deprivation, and multiple medical comorbidities. ${ }^{53,54}$

Detection of delirium in the ICU is of paramount importance. A number of tools to screen and diagnose delirium at the bedside have been developed. The two most commonly used and validated instruments are the Intensive Care Delirium Screening Checklist (ICDSC) ${ }^{55}$ and the Confusion Assessment Method for the Intensive Care Unit (CAM-ICU) (Fig. 5). Both the ICDSC and the CAM-ICU have been shown to be sensitive in the detection of delirium compared to the criteria set out in the Diagnostic and Statistical Manual of Mental Disorders. ${ }^{56}$ The CAM-ICU demonstrates greater specificity for the diagnosis of delirium $^{57}$ and consists of four components: (1) acute onset of mental status change or fluctuating course, (2) evidence of inattention, (3) evidence of disorganized thinking, and (4) altered level of consciousness (Fig. 5). Delirium is diagnosed when a patient exhibits components 1 and 2 in addition to either component 3 or $4 .{ }^{58}$ Delirium screening with one of the above tools should occur daily in the ICU, and ideally multiple times throughout the day. 
Pharmacologic prevention of delirium remains an active area of investigation, although routine use of pharmacologic agents in all critically ill adults is not recommended in the 2018 PADIS Guidelines. The randomized, doubleblind, placebo-controlled REDUCE trial investigated whether prophylactic use of haloperidol would improve survival or reduce delirium incidence and increase delirium- and coma-free days. It showed no improved survival in the intervention group and no statistical difference in any of the delirium outcomes compared to placebo. ${ }^{59}$ Work by $\mathrm{Su}$ and colleagues ${ }^{60}$ suggested that dexmedetomidine prevented delirium in elderly adults following non-cardiac surgery, although this work involved a low severity of illness in a postoperative patient population that limited its generalizability to most critically ill patients. A similar trial evaluated whether the use of low-dose nocturnal infusions of dexmedetomidine prevented delirium; the authors reported that the proportion of patients who remained free of delirium during their ICU admission was greater in the intervention group. ${ }^{61}$ Limitations of that study included its sample size and the limited number of total deliriumfree days. Other agents, such as melatonin, have sparked interest but data regarding efficacy are limited. Currently, the PADIS guidelines do not recommend pharmacologic prevention of delirium and instead recommend a focus on improving sleep hygiene, practicing early mobilization, and improving the ICU environment to help prevent the development of delirium.

Management of delirium once it has developed should focus on the identification of any potentially modifiable precipitating factors, such as unrecognized infection or untreated disease, removing offending drugs, and optimizing the patient environment by promoting sleep, reducing noise, practicing early mobilization, and providing needed assistive devices such as hearing aids and eye glasses. ${ }^{62,63}$ The respiratory therapist will often note evidence of pain and patient-ventilator asynchrony, which could be signs of delirium as well as precipitating factors for its development. Treatment of the underlying critical illness remains the foundation for delirium resolution because there are few pharmacologic management options to treat delirium after it develops. Antipsychotics have traditionally been used to treat delirium, but there is no definitive evidence that these prevent or reduce the duration of delirium. ${ }^{64-66}$ The recent MIND-USA trial, a multi-center, double-blind, randomized, placebo-controlled trial of haloperidol and ziprasidone versus placebo in the treatment of delirium during critical illness, reported no difference in median days alive without delirium or coma. ${ }^{67}$ There were also no other significant differences in median days to ICU discharge, median days to freedom from mechanical ventilation, or 30- and 90-d mortality. The mounting evidence shows that the treatment of delirium with antipsychotics is

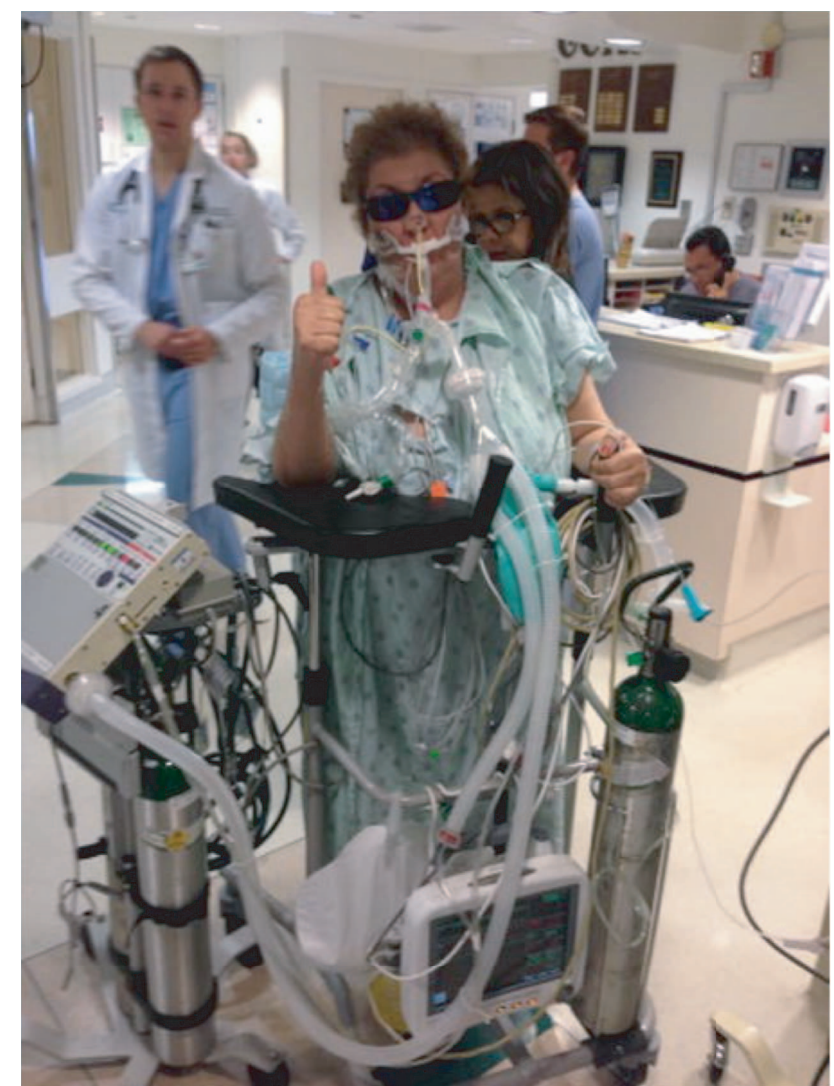

Fig. 6. Mobilizing an intubated patient with advanced equipment including monitors and pumps. This patient provides a visual example of the incorporation of the ABCDEF bundle. Courtesy Heidi Engel and University of California San Franscisco.

not effective and that other potential therapeutic options need further exploration.

\section{Early Mobility and Exercise}

Early mobilization is an important but often underutilized component of the ABCDEF bundle. Critically ill patients are frequently bed-bound and immobile for days due to their illness, sedation, and hemodynamic instability. Often, there is significant concern from the care team regarding the safety of early mobilization and physical activity in ICU patients; however, mobilization has been demonstrated to be safe, even in patients receiving invasive support such as mechanical ventilation, renal replacement therapy, and extracorporeal membrane oxygenation cardiopulmonary support (Fig. 6). ${ }^{68-70}$ Prolonged immobilization results in muscle wasting and weakness, as well as the development of ICU-acquired weakness, leading to poor functional status, disability, and reduced exercise capacity in ICU survivors for years following their illness. ${ }^{71-74}$

RTs are integral in assisting patients with early mobilization, particularly patients receiving significant respira- 
tory support, and can advocate for early mobility based on the evidence of safety and benefit. Schweickert and colleagues $^{75}$ demonstrated that early (ie, $<3 \mathrm{~d}$ of mechanical ventilation) physical and occupational therapy and mobilization, paired with a daily SAT, reduced the duration of delirium and improved functional status at discharge with minimal risk. Notably, early mobilization has been the only intervention shown to decrease the number of days of delirium. ${ }^{76}$ Mobilizing patients early in their ICU course has also been shown to be safe and is associated with decreased ICU and hospital length of stay. ${ }^{77}$ Another study with later initiation of physical therapy and mobilization, however, did not demonstrate any improvement in longterm physical functioning, ${ }^{78}$ suggesting that mobilization early in the course of critical illness is important, although this was not corroborated in a similar study of early mobilization by Morris and colleagues. ${ }^{79}$ Taken together, all of these studies suggest that mobilization of critically ill patients, including those on mechanical ventilation, can be done safely and can improve long-term outcomes.

Early mobility is an integral part of the ABCDEF bundle of care, and increasing compliance with individual components of the bundle has demonstrated substantial improvements in mortality, delirium duration, ICU and hospital length of stay, and days free from mechanical ventilation. ${ }^{11,80}$ Given this, early mobility should be advocated as a part of the routine care of the critically ill patient.

\section{Family Engagement and Empowerment}

In the ICU, patients are often unable to communicate with their care team and their families due to their underlying illness and medical interventions. Patient-centered care respects individual patient values and informs clinical decision making. Failing to engage family members and adequately communicate represents a significant missed opportunity to identify treatment preferences that are in line with the values of the patient and that respect their inherent dignity and value. ${ }^{81}$ In the ICU setting, empowering family members to engage in shared decision making and facilitating open communication is of paramount importance.

Critical illness can lead to significant psychological burden for not only patients but also their families. ${ }^{5,82-84}$ The ABCDEF bundle has grown to include family engagement and empowerment for this very reason because fully engaging families during this time gives voice to patients and their loved ones. Heyland and colleagues ${ }^{85}$ showed that family satisfaction with medical care is higher when they are engaged and involved in their loved one's care, and clinician-facilitated support during family conferences also improved satisfaction with care and communication. ${ }^{86}$ Family presence during rounds also improved some families'
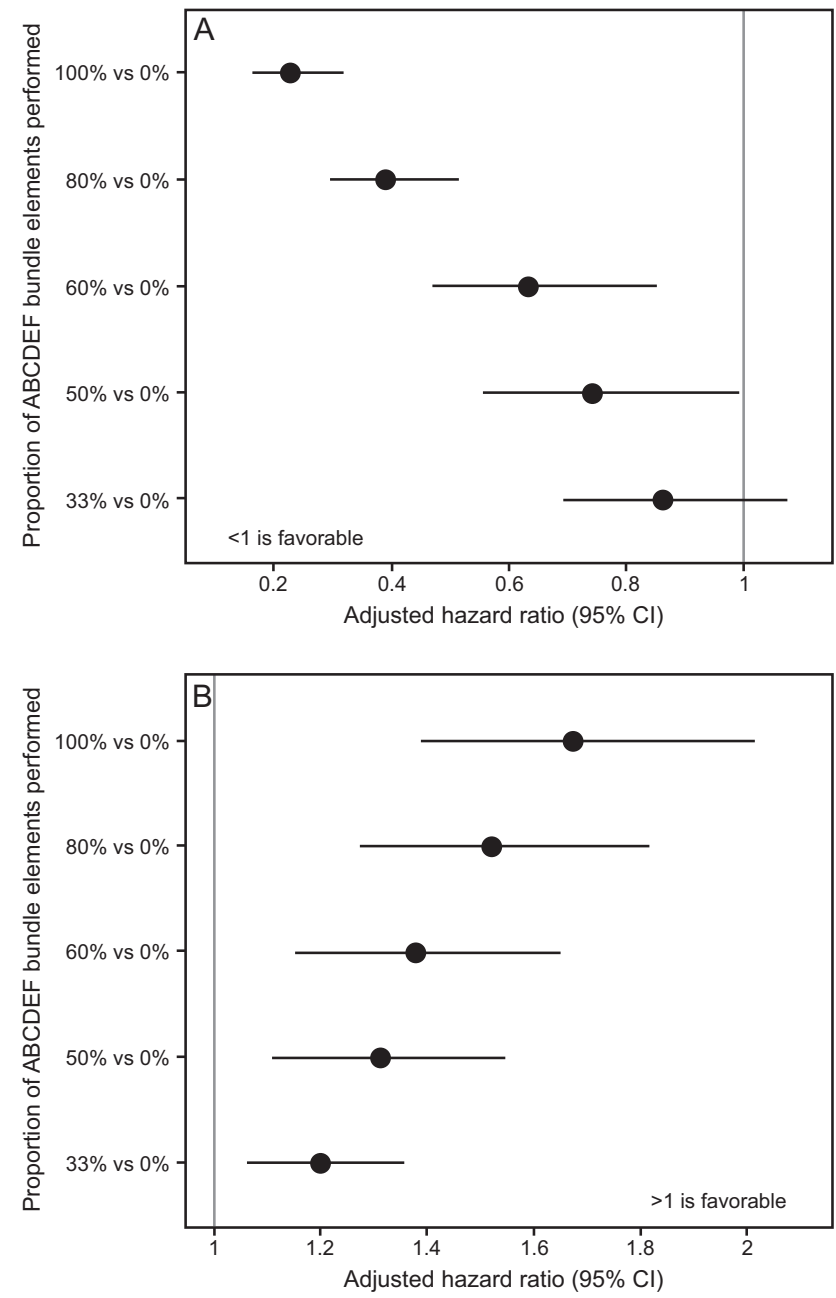

Fig. 7. Association between proportional ABCDEF bundle performance and mortality and ICU discharge. From Reference 11, with permission.

impression of communication and understanding of the patients' care. ${ }^{87}$ Although a study evaluating a directed family-support intervention for surrogates of patients with critical illness did not show a reduction in psychological distress in family members, it did increase families' perception of quality communication and patient and family centered care as well as reduce ICU length of stay. ${ }^{88}$ Studies of ICU diaries, where patients or their families write down daily thoughts and memories of their ICU stay, have been associated with reduced symptoms of post-traumatic stress disorder in patients and families. ${ }^{89,90}$ For patients who ultimately have illnesses that are not survivable, focused communication with family members through conferences and palliative care or ethics consultation facilitated the transition of care goals to palliative and comfort-focused measures and limited a protracted dying process. ${ }^{91-93}$ Additionally, family presence during cardiopulmonary resuscitation has been associated with a re- 

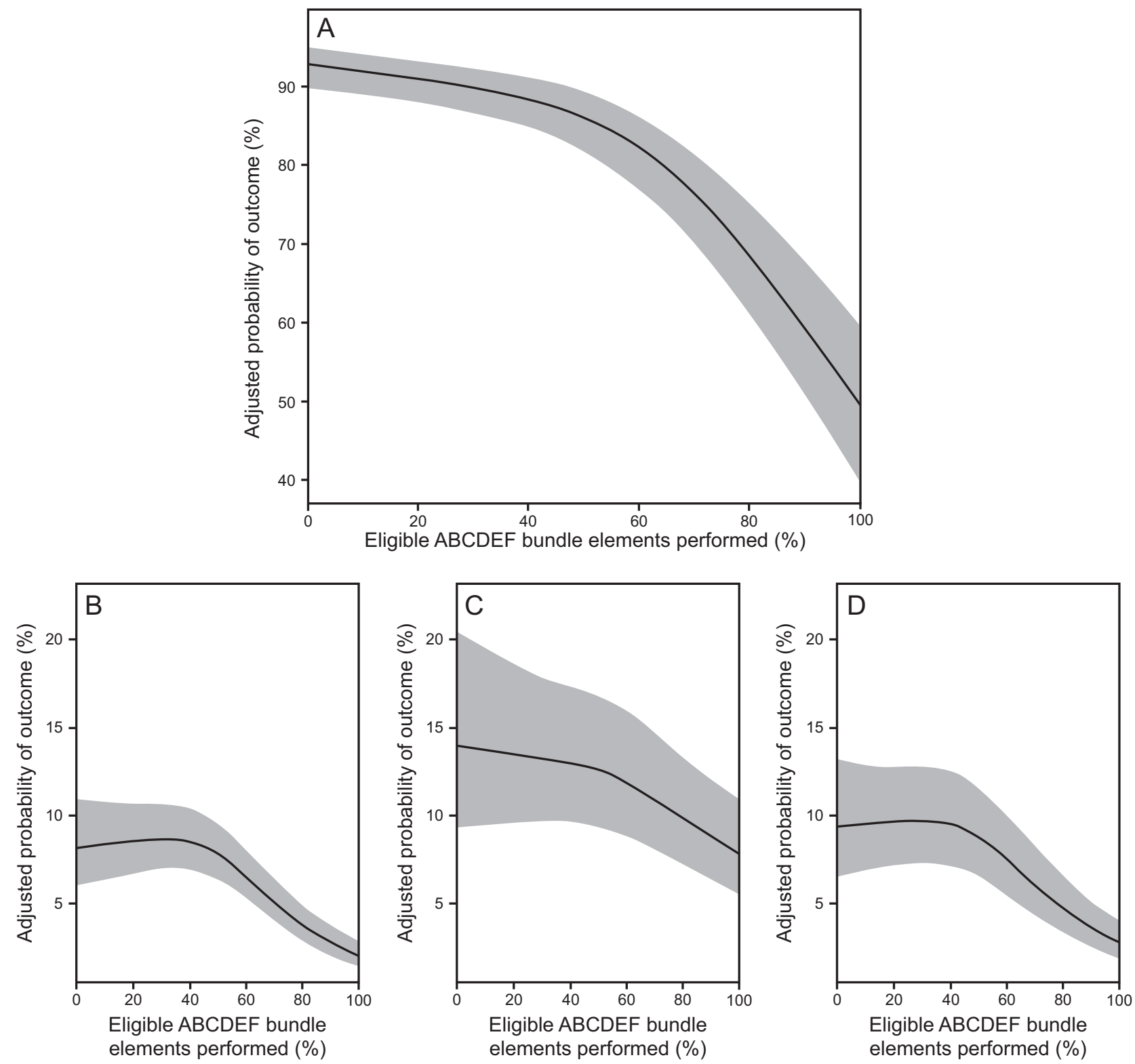

Fig. 8. Association between proportional performance of the ABCDEF bundle and (A) next-day mechanical ventilation, (B) coma, (C) delirium, and (D) need for physical restraints. From Reference 11, with permission.

duced incidence of symptoms of anxiety and depression as compared to those who were not present during the resuscitation efforts. ${ }^{94}$

Family engagement and empowerment in the ICU is multidisciplinary and necessitates input from multiple clinicians, including RTs. Providing information regarding the role of each team member, answering questions regarding diagnosis and treatment, and engaging the family in the care of their loved one facilitates a supportive environment and can reduce the stress of acute illness. The experience of critical illness reaches beyond just the patient to family members and loved ones who continue in supportive roles. The ABCDEF bundle, by including fam- ily engagement, helps build a relationship of trust between patients, families, and their medical team that facilitates communication and respect and puts patients and their values at the forefront of care.

\section{The ABCDEF Bundle: Synergistic Care Improving Outcomes}

Combining processes of care together into a "bundle" is an oft-utilized tactic in medicine, and each of the individual components of the ABCDEF bundle have demonstrated benefit in multiple peer-reviewed studies in critically ill subjects. In the ICU, the track record of bundles in im- 
proving outcomes is mixed, however. Sepsis bundles and central venous catheter bundles have been successful in reducing mortality and complications from common ICU interventions. $.^{95}, 96$ However, a complex quality-improvement bundle including low tidal volume ventilation, moderate sedation, venous thromboembolism prophylaxis, nutrition, head of bed elevation, and urinary and central venous catheter use in 118 Brazilian ICUs did not change mortality or improve patient outcomes. ${ }^{97}$ Each of these assorted interventions have been shown to be beneficial, but when combined into a larger, more complicated initiative, outcomes did not change. Although the reasons this particular bundle may not improve outcomes are complex, one important reason is that the individual interventions were not necessarily symbiotic.

The ABCDEF bundle is multi-faceted, but each of the individual components are synergistic and linked. For example, managing pain and achieving light sedation in a critically ill, mechanically ventilated patient helps prevent delirium but also facilitates early mobility as well as helps the patient be more interactive with family. The importance of this synergy has been demonstrated in 2 large studies. In a large quality-improvement study across 7 community hospitals in California, Barnes-Daly et al ${ }^{80}$ showed that compliance with the ABCDEF bundle, both total and partial, was associated with decreased mortality and an increase in delirium- and coma-free days independent of severity of illness and age (Fig. 1). Notably, the study showed that, for every $10 \%$ increase in partial bundle compliance, there was a $7 \%$ higher odds of hospital survival. In a seminal study of $>15,000$ subjects across 68 academic, community, and federal ICUs, performance of the ABCDEF bundle was associated with multiple improved outcomes, including hospital death within $7 \mathrm{~d}$, days of mechanical ventilation, days of delirium and coma, restraint use, ICU readmission, and discharge to a facility (Fig. 7 and Fig. 8). ${ }^{11}$ In this study, outcomes were related to the proportion of bundle performance in a dose-dependent manner, with higher proportions of bundle performance correlating with better outcomes. These results demonstrate the benefit of bundling and coordinating interdependent processes of care that address the complex nature of critical illness and highlight the importance of a multidisciplinary approach.

RTs are uniquely positioned to ensure the successful implementation of the ABCDEF bundle. Routine assessment of readiness for extubation through performance of SBTs, communicating evidence of pain or ventilator asynchrony to the bedside nurses, enabling early mobilization, and educating families on what to expect while patients are receiving respiratory support are all crucial elements related to the ABCDEF bundle. RTs also facilitate communication between physicians, nurses, physical therapists, and other members of the care team. Additionally, utiliz- ing a multi-layered process of care can have barriers to implementation, ${ }^{98-100}$ so understanding the individual elements, the impact that the ABCDEF bundle has in the care of critically ill patients, and the unique culture and organization of ICUs puts RTs in an instrumental position to implement best practices and provide insight into the necessary requirements for building the bundle in their own institutions.

\section{Conclusions and Future Directions}

In the last several decades, the care of the critically ill patient has morphed from one of deep sedation, limited mobility, and minimal family engagement to one of light sedation, early mobility, recognition and management of delirium, and increasing family empowerment. These changes came in response to the recognition of the longterm consequences of critical illness and the impact of medical interventions routinely performed in the ICU. The $\mathrm{ABCDEF}$ bundle has been developed over time to address these needs, and it is improving the care of our sickest patients, but there is still more work to be done. ${ }^{101}$ Future research questions moving forward are numerous but include identifying optimum sedation agents for patients on mechanical ventilation both with and without delirium, optimizing implementation in ICUs with varying amounts of resources, and identifying strategies to reduce family and caregiver distress during and after critical illness. RTs are a vital link in the implementation the ABCDEF bundle and in promoting culture change, which will continue to be crucial to the success of critical care medicine in the years ahead.

\section{REFERENCES}

1. Mullins PM, Goyal M, Pines JM. National growth in intensive care unit admissions from emergency departments in the United States from 2002 to 2009. Acad Emerg Med 2013;20(5):479-486.

2. Ely EW. The ABCDEF bundle: science and philosophy of how ICU liberation serves patients and families. Crit Care Med 2017;45(2): 321-330.

3. Pandharipande PP, Girard TD, Jackson JC, Morandi A, Thompson JL, Pun BT, et al. Long-term cognitive impairment after critical illness. N Engl J Med 2013;369(14):1306-1316.

4. Brummel NE, Bell SP, Girard TD, Pandharipande PP, Jackson JC, Morandi A, et al. Frailty and subsequent disability and mortality among patients with critical illness. Am J Respir Crit Care Med 2016;196(1):64-72.

5. Jackson JC, Pandharipande PP, Girard TD, Brummel NE, Thompson JL, Hughes CG, et al. Depression, post-traumatic stress disorder, and functional disability in survivors of critical illness in the BRAIN-ICU study: a longitudinal cohort study. Lancet Respir Med 2014;2(5):369-379.

6. Vasilevskis EE, Ely EW, Speroff T, Pun BT, Boehm L, Dittus RS. Reducing iatrogenic risks ICU-acquired delirium and weaknesscrossing the quality chasm. Chest 2010;138(5):1224-1233.

7. Barr J, Fraser GL, Puntillo K, Ely EW, Gelinas C, Dasta JF, et al. Clinical practice guidelines for the management of pain, agitation, 
and delirium in adult patients in the intensive care unit. Crit Care Med 2013;41(1):263-306.

8. Devlin JW, Skrobik Y, Gelinas C, Needham DM, Slooter AJC, Pandharipande PP, et al. Clinical practice guidelines for the prevention and management of pain, agitation/sedation, delirium, immobility, and sleep disruption in adult patients in the ICU. Crit Care Med 2018;46(9):e825-e873.

9. Pandharipande P, Banerjee A, McGrane S, Ely EW. Liberation and animation for ventilated ICU patients: the ABCDE bundle for the back-end of critical care. Crit Care 2010;14(3):157.

10. Morandi A, Brummel NE, Ely EW. Sedation, delirium and mechanical ventilation: the 'ABCDE' approach. Curr Opin Crit Care 2011;17(1):43-49.

11. Pun BT, Balas MC, Barnes-Daly MA, Thompson JL, Aldrich JM, Barr J, et al. Caring for critically ill patients with the ABCDEF bundle: results of the ICU liberation collaborative in over 15,000 adults. Crit Care Med 2019;47(1):3-14.

12. Chanques G, Viel E, Constantin JM, Jung B, de Lattre S, Carr J, et al. The measurement of pain in intensive care unit: comparison of 5 self-report intensity scales. Pain 2010;151(3):711-721.

13. Payen JF, Chanques G, Mantz J, Hercule C, Auriant I, Leguillou $\mathrm{JL}$, et al. Current practices in sedation and analgesia for mechanically ventilated critically ill patients: a prospective multicenter patient-based study. Anesthesiology 2007;106(4):687-695.

14. Puntillo KA, Max A, Timsit JF, Vignoud L, Chanques G, Robleda $\mathrm{G}$, et al. Determinants of procedural pain intensity in the intensive care unit. The Europain(R) study. Am J Respir Crit Care Med 2014;189(1):39-47.

15. Robinson S, Vollmer C, Jirka H, Rich C, Midiri C, Bisby D. Aging and delirium: too much or too little pain medication? Pain Manag Nurs 2008;9(2):66-72.

16. Nie H, Zhao B, Zhang Y-Q, Jiang Y-H, Yang Y-X. Pain and cognitive dysfunction are the risk factors of delirium in elderly hip fracture Chinese patients. Arch Gerontol Geriatr 2012;54(2):e172e174.

17. Payen JF, Bru O, Bosson JL, Lagrasta A, Novel E, Deschaux I, et al. Assessing pain in critically ill sedated patients by using a behavioral pain scale. Crit Care Med 2001;29(12):2258-2263.

18. Gelinas C, Johnston C. Pain assessment in the critically ill ventilated adult: validation of the critical-care pain observation tool and physiologic indicators. Clin J Pain 2007;23(6):497-505.

19. Gelinas C, Fillion L, Puntillo KA, Viens C, Fortier M. Validation of the critical-care pain observation tool in adult patients. Am J Crit Care 2006;15(4):420-427.

20. Gelinas C. Nurses' evaluations of the feasibility and the clinical utility of the critical-care pain observation tool. Pain Manag Nurs 2010;11(2):115-125.

21. Gelinas C, Arbour C, Michaud C, Vaillant F, Desjardins S. Implementation of the critical-care pain observation tool on pain assessment/management nursing practices in an intensive care unit with nonverbal critically ill adults: a before and after study. Int J Nurs Stud 2011;48(12):1495-1504.

22. Payen JF, Bosson JL, Chanques G, Mantz J, Labarere J, Investigators D. Pain assessment is associated with decreased duration of mechanical ventilation in the intensive care unit: a post hoc analysis of the DOLOREA study. Anesthesiology 2009;111(6):1308-1316.

23. Kress JP, Pohlman AS, O'Connor MF, Hall JB. Daily interruption of sedative infusions in critically ill patients undergoing mechanical ventilation. N Engl J Med 2000;342(20):1471-1477.

24. Kress JP, Gehlbach B, Lacy M, Pliskin N, Pohlman AS, Hall JB. The long-term psychological effects of daily sedative interruption on critically ill patients. Am J Respir Crit Care Med 2003;168(12): $1457-1461$.
25. Schweickert WD, Gehlbach BK, Pohlman AS, Hall JB, Kress JP. Daily interruption of sedative infusions and complications of critical illness in mechanically ventilated patients. Crit Care Med 2004; 32(6):1272-1276.

26. Shehabi Y, Bellomo R, Reade MC, Bailey M, Bass F, Howe B, et al. Early intensive care sedation predicts long-term mortality in ventilated critically ill patients. Am J Respir Crit Care Med 2012; 186(8):724-731.

27. Shehabi Y, Chan L, Kadiman S, Alias A, Ismail WN, Tan MA, et al. Sedation depth and long-term mortality in mechanically ventilated critically ill adults: a prospective longitudinal multicentre cohort study. Intensive Care Med 2013;39(5):910-918.

28. Esteban A, Alia I, Gordo F, Fernandez R, Solsona JF, Vallverdu I, et al. Extubation outcome after spontaneous breathing trials with T-tube or pressure support ventilation. The Spanish Lung Failure Collaborative Group. Am J Respir Crit Care Med 156(2 Pt 1):459465, 1997.

29. Ely EW, Baker AM, Dunagan DP, Burke HL, Smith AC, Kelly PT, et al. Effect on the duration of mechanical ventilation of identifying patients capable of breathing spontaneously. N Engl J Med 1996; 335(25):1864-1869.

30. Girard TD, Kress JP, Fuchs BD, Thomason JW, Schweickert WD, Pun BT, et al. Efficacy and safety of a paired sedation and ventilator weaning protocol for mechanically ventilated patients in intensive care (awakening and breathing controlled trial): a randomised controlled trial. Lancet 2008;371(9607):126-134.

31. Esteban A, Alia I, Tobin MJ, Gil A, Gordo F, Vallverdu I, et al. Effect of spontaneous breathing trial duration on outcome of attempts to discontinue mechanical ventilation. Spanish Lung Failure Collaborative Group. Am J Respir Crit Care Med 1999;159(2):512518.

32. Khan BA, Guzman O, Campbell NL, Walroth T, Tricker J, Hui SL, et al. Comparison and agreement between the Richmond AgitationSedation Scale and the Riker Sedation-Agitation Scale in evaluating patients' eligibility for delirium assessment in the ICU. Chest 2012;142(1):48-54.

33. Ely EW, Truman B, Shintani A, Thomason JW, Wheeler AP, Gordon S, et al. Monitoring sedation status over time in ICU patients: reliability and validity of the Richmond Agitation-Sedation Scale (RASS). JAMA 2003;289(22):2983-2991.

34. Treggiari MM, Romand JA, Yanez ND, Deem SA, Goldberg J, Hudson L, et al. Randomized trial of light versus deep sedation on mental health after critical illness. Crit Care Med 2009;37(9):25272534.

35. Shehabi Y, Bellomo R, Reade MC, Bailey M, Bass F, Howe B, et al. Early goal-directed sedation versus standard sedation in mechanically ventilated critically ill patients: a pilot study. Crit Care Med 2013;41(8):1983-1991.

36. Bugedo G, Tobar E, Aguirre M, Gonzalez H, Godoy J, Lira MT, et al. The implementation of an analgesia-based sedation protocol reduced deep sedation and proved to be safe and feasible in patients on mechanical ventilation. Rev Bras Ter Intensiva 2013;25(3):188196.

37. Shehabi Y, Bellomo R, Kadiman S, Ti LK, Howe B, Reade MC, et al. Sedation intensity in the first 48 hours of mechanical ventilation and 180-day mortality: a multinational prospective longitudinal cohort study. Crit Care Med 2018;46(6):850-859.

38. Kollef MH, Levy NT, Ahrens TS, Schaiff R, Prentice D, Sherman G. The use of continuous IV sedation is associated with prolongation of mechanical ventilation. Chest 1998;114(2):541-548.

39. Dale CR, Kannas DA, Fan VS, Daniel SL, Deem S, Yanez ND 3rd, et al. Improved analgesia, sedation, and delirium protocol associated with decreased duration of delirium and mechanical ventilation. Ann Am Thorac Soc 2014;11(3):367-374. 
40. Pandharipande P, Shintani A, Peterson J, Pun BT, Wilkinson GR, Dittus RS, et al. Lorazepam is an independent risk factor for transitioning to delirium in intensive care unit patients. Anesthesiology 2006;104(1):21-26.

41. Seymour CW, Pandharipande PP, Koestner T, Hudson LD, Thompson JL, Shintani AK, et al. Diurnal sedative changes during intensive care: impact on liberation from mechanical ventilation and delirium. Crit Care Med 2012;40(10):2788-2796.

42. Pandharipande PP, Pun BT, Herr DL, Maze M, Girard TD, Miller $\mathrm{RR}$, et al. Effect of sedation with dexmedetomidine vs lorazepam on acute brain dysfunction in mechanically ventilated patients: the MENDS randomized controlled trial. JAMA 2007;298(22):26442653.

43. Riker RR, Shehabi Y, Bokesch PM, Ceraso D, Wisemandle W, Koura F, et al. Dexmedetomidine vs midazolam for sedation of critically ill patients: a randomized trial. JAMA 2009;301(5):489499.

44. Grounds RM, Lalor JM, Lumley J, Royston D, Morgan M. Propofol infusion for sedation in the intensive care unit: preliminary report. BMJ (Clin Res Ed) 1987;294(6569):397-400.

45. Roekaerts PM, Huygen FJ, de Lange S. Infusion of propofol versus midazolam for sedation in the intensive care unit following coronary artery surgery. J Cardiothorac Vasc Anesth 1993;7(2):142147.

46. McMurray TJ, Collier PS, Carson IW, Lyons SM, Elliott P. Propofol sedation after open heart surgery: a clinical and pharmacokinetic study. Anaesthesia 1990;45(4):322-326.

47. Jakob SM, Ruokonen E, Grounds RM, Sarapohja T, Garratt C, Pocock SJ, et al. Dexmedetomidine vs midazolam or propofol for sedation during prolonged mechanical ventilation: two randomized controlled trials. JAMA 2012;307(11):1151-1160.

48. American Psychiatric Association. Diagnostic and statistical manual of mental disorders: DSM-5. Washington DC: American Psychiatric Association; 2013.

49. Pun BT, Ely EW. The importance of diagnosing and managing ICU delirium. Chest 2007;132(2):624-636.

50. Ely EW, Shintani A, Truman B, Speroff T, Gordon SM, Harrell FE $\mathrm{Jr}$, et al. Delirium as a predictor of mortality in mechanically ventilated patients in the intensive care unit. JAMA 2004;291(14): 1753-1762.

51. Lin SM, Liu CY, Wang CH, Lin HC, Huang CD, Huang PY, et al. The impact of delirium on the survival of mechanically ventilated patients. Crit Care Med 2004;32(11):2254-2259.

52. Jackson JC, Hart RP, Gordon SM, Shintani A, Truman B, May L, et al. Six-month neuropsychological outcome of medical intensive care unit patients. Crit Care Med 2003;31(4):1226-1234.

53. Inouye SK, Viscoli CM, Horwitz RI, Hurst LD, Tinetti ME. A predictive model for delirium in hospitalized elderly medical patients based on admission characteristics. Ann Intern Med 1993; 119(6):474-481.

54. Girard TD, Thompson JL, Pandharipande PP, Brummel NE, Jackson JC, Patel MB, et al. Clinical phenotypes of delirium during critical illness and severity of subsequent long-term cognitive impairment: a prospective cohort study. Lancet Respir Med 2018;6(3): 213-222.

55. Bergeron N, Dubois MJ, Dumont M, Dial S, Skrobik Y. Intensive Care Delirium Screening Checklist: evaluation of a new screening tool. Intensive Care Med 2001;27(5):859-864.

56. Gusmao-Flores D, Salluh JI, Chalhub RA, Quarantini LC. The confusion assessment method for the intensive care unit (CAMICU) and intensive care delirium screening checklist (ICDSC) for the diagnosis of delirium: a systematic review and meta-analysis of clinical studies. Crit Care 2012;16(4):R115.
57. Reade MC, Finfer S. Sedation and delirium in the intensive care unit. N Engl J Med 2014;370(5):444-454.

58. Ely EW, Inouye SK, Bernard GR, Gordon S, Francis J, May L, et al. Delirium in mechanically ventilated patients: validity and reliability of the confusion assessment method for the intensive care unit (CAM-ICU). JAMA 2001;286(21):2703-2710.

59. van den Boogaard M, Slooter AJC, Bruggemann RJM, Schoonhoven L, Beishuizen A, Vermeijden JW, et al. Effect of haloperidol on survival among critically ill adults with a high risk of delirium: the REDUCE randomized clinical trial. JAMA 2018;319(7):680690.

60. Su X, Meng ZT, Wu XH, Cui F, Li HL, Wang DX, et al. Dexmedetomidine for prevention of delirium in elderly patients after noncardiac surgery: a randomised, double-blind, placebo-controlled trial. Lancet 2016;388(10054):1893-1902.

61. Skrobik Y, Duprey MS, Hill NS, Devlin JW. Low-dose nocturnal dexmedetomidine prevents ICU delirium: a randomized, placebocontrolled trial. Am J Respir Crit Care Med 2018;197(9):11471156.

62. Ely EW, Siegel MD, Inouye SK. Delirium in the intensive care unit: an under-recognized syndrome of organ dysfunction. Semin Respir Crit Care Med 2001;22(2):115-126.

63. Ely EW, Gautam S, Margolin R, Francis J, May L, Speroff T, et al. The impact of delirium in the intensive care unit on hospital length of stay. Intensive Care Med 2001;27(12):1892-1900.

64. Girard TD, Pandharipande PP, Carson SS, Schmidt GA, Wright PE, Canonico AE, et al. Feasibility, efficacy, and safety of antipsychotics for intensive care unit delirium: the MIND randomized, placebo-controlled trial. Crit Care Med 2010;38(2):428-437.

65. Neufeld KJ, Yue J, Robinson TN, Inouye SK, Needham DM. Antipsychotic medication for prevention and treatment of delirium in hospitalized adults: a systematic review and meta-analysis. J Am Geriatr Soc 2016;64(4):705-714.

66. Page VJ, Ely EW, Gates S, Zhao XB, Alce T, Shintani A, et al. Effect of intravenous haloperidol on the duration of delirium and coma in critically ill patients (Hope-ICU): a randomised, doubleblind, placebo-controlled trial. Lancet Respir Med 2013;1(7):515523.

67. Girard TD, Exline MC, Carson SS, Hough CL, Rock P, Gong MN, et al. Haloperidol and ziprasidone for treatment of delirium in critical illness. N Engl J Med 2018;379(26):2506-2516.

68. Bailey P, Thomsen GE, Spuhler VJ. Early activity is feasible and safe in respiratory failure patients. Crit Care Med 2007;35(1):139145.

69. Dammeyer J, Dickinson S, Packard D, Baldwin N, Ricklemann C. Building a protocol to guide mobility in the ICU. Crit Care Nurs Q 2013;36(1):37-49.

70. Freeman R, Maley K. Mobilization of intensive care cardiac surgery patients on mechanical circulatory support. Crit Care Nurs Q 2013;36(1):73-88.

71. Puthucheary ZA, Rawal J, McPhail M, Connolly B, Ratnayake G, Chan $\mathrm{P}$, et al. Acute skeletal muscle wasting in critical illness. JAMA 2013;310(15):1591-1600

72. Morris PE. Moving our critically ill patients: mobility barriers and benefits. Crit Care Clin 2007;23(1):1-20.

73. Herridge MS, Cheung AM, Tansey CM, Matte-Martyn A, DiazGranados N, Al-Saidi F, et al. One-year outcomes in survivors of the acute respiratory distress syndrome. N Engl J Med 2003;348(8): 683-693.

74. Herridge MS, Tansey CM, Matte A, Tomlinson G, Diaz-Granados N, Cooper A, et al. Functional disability 5 years after acute respiratory distress syndrome. N Engl J Med 2011;364(14):1293-1304.

75. Schweickert WD, Pohlman MC, Pohlman AS, Nigos C, Pawlik AJ, Esbrook CL, et al. Early physical and occupational therapy in me- 
chanically ventilated, critically ill patients: a randomised controlled trial. Lancet 2009;373(9678):1874-1882.

76. Kress JP, Hall JB. ICU-acquired weakness and recovery from critical illness. N Engl J Med 2014;370(17):1626-1635.

77. Morris PE, Goad A, Thompson C. Early intensive care unit mobility therapy in the treatment of acute respiratory failure. Crit Care Med 2008;36(8):2238-2243.

78. Moss M, Nordon-Craft A, Malone D, Van Pelt D, Frankel SK, Warner ML, et al. A randomized trial of an intensive physical therapy program for acute respiratory failure patients. Am J Respir Crit Care Med 2015;193(10):1101-1110.

79. Morris PE, Berry MJ, Files DC, Thompson JC, Hauser J, Flores L, et al. Standardized rehabilitation and hospital length of stay among patients with acute respiratory failure: a randomized clinical trial. JAMA 2016;315(24):2694-2702.

80. Barnes-Daly MA, Phillips G, Ely EW. Improving hospital survival and reducing brain dysfunction at seven california community hospitals: implementing PAD guidelines via the $A B C D E F$ bundle in 6,064 patients. Crit Care Med 2017;45(2):171-178.

81. Curtis JR, Engelberg RA, Wenrich MD, Shannon SE, Treece PD, Rubenfeld GD. Missed opportunities during family conferences about end-of-life care in the intensive care unit. Am J Respir Crit Care Med 2005;171(8):844-849.

82. Jackson JC, Mitchell N, Hopkins RO. Cognitive functioning, mental health, and quality of life in ICU survivors: an overview. Psychiatr Clin North Am 2015;38(1):91-104.

83. Huang M, Parker AM, Bienvenu OJ, Dinglas VD, Colantuoni E, Hopkins RO, et al. Psychiatric symptoms in acute respiratory distress syndrome survivors: a 1-year national multicenter study. Crit Care Med 2016;44(5):954-965.

84. Azoulay E, Pochard F, Chevret S, Arich C, Brivet F, Brun F, et al. Family participation in care to the critically ill: opinion of families and staff. Intensive Care Med 2003;29:1498-1504.

85. Heyland DK, Rocker GM, Dodek PM, Kutsogiannis DJ, Konopad E, Cook DJ, et al. Family satisfaction with care in the intensive care unit: results of a multiple center study. Crit Care Med 2002;30(7): 1413-1418.

86. Stapleton RD, Engelberg RA, Wenrich MD, Goss CH, Curtis JR. Clinician statements and family satisfaction with family conferences in the intensive care unit. Crit Care Med 2006;34(6):16791685 .

87. Jacobowski NL, Girard TD, Mulder JA, Ely EW. Communication in critical care: family rounds in the intensive care unit. Am J Crit Care 2010;19(5):421-430.

88. White DB, Angus DC, Shields AM, Buddadhumaruk P, Pidro C, Paner C, et al. A randomized trial of a family-support intervention in intensive care units. N Engl J Med 2018;378(25):2365-2375.

89. Jones C, Backman C, Capuzzo M, Egerod I, Flaatten H, Granja C, et al. Intensive care diaries reduce new onset post traumatic stress disorder following critical illness: a randomised, controlled trial. Crit Care 2010;14(5):R168
90. Jones C, Backman C, Griffiths RD. Intensive care diaries and relatives' symptoms of posttraumatic stress disorder after critical illness: a pilot study. Am J Crit Care 2012;21(3):172-176.

91. Campbell ML, Guzman JA. Impact of a proactive approach to improve end-of-life care in a medical ICU. Chest 2003;123(1): 266-271.

92. Schneiderman LJ, Gilmer T, Teetzel HD, Dugan DO, Blustein J, Cranford R, et al. Effect of ethics consultations on nonbeneficial life-sustaining treatments in the intensive care setting: a randomized controlled trial. JAMA 2003;290(9):1166-1172.

93. Lilly CM, De Meo DL, Sonna LA, Haley KJ, Massaro AF, Wallace $\mathrm{RF}$, et al. An intensive communication intervention for the critically ill. Am J Med 2000;109(6):469-475.

94. Jabre P, Belpomme V, Azoulay E, Jacob L, Bertrand L, Lapostolle $F$, et al. Family presence during cardiopulmonary resuscitation. N Engl J Med 2013;368(11):1008-1018.

95. Levy MM, Dellinger RP, Townsend SR, Linde-Zwirble WT, Marshall JC, Bion J, et al. The Surviving Sepsis Campaign: results of an international guideline-based performance improvement program targeting severe sepsis. Crit Care Med 2010;38(2):367-374.

96. Pronovost P, Needham D, Berenholtz S, Sinopoli D, Chu H, Cosgrove $\mathrm{S}$, et al. An intervention to decrease catheter-related bloodstream infections in the ICU. N Engl J Med 2006;355(26):27252732.

97. Cavalcanti AB, Bozza FA, Machado FR, Salluh JI, Campagnucci VP, Vendramim P, et al. Effect of a quality improvement intervention with daily round checklists, goal setting, and clinician prompting on mortality of critically ill patients: a randomized clinical trial. JAMA 2016;315(14):1480-1490.

98. Carrothers KM, Barr J, Spurlock B, Ridgely MS, Damberg CL, Ely EW. Contextual issues influencing implementation and outcomes associated with an integrated approach to managing pain, agitation, and delirium in adult ICUs. Crit Care Med 2013;41(9 Suppl 1): S128-S135.

99. Boehm LM, Vasilevskis EE, Dietrich MS, Wells N, Ely EW, Pandharipande $\mathrm{P}$, et al. Organizational domains and variation in attitudes of intensive care providers toward the ABCDE bundle. Am J Crit Care 2017;26(3):e18-e28.

100. Boehm LM, Dietrich MS, Vasilevskis EE, Wells N, Pandharipande P, Ely EW, et al. Perceptions of workload burden and adherence to $\mathrm{ABCDE}$ bundle among intensive care providers. Am J Crit Care 2017;26(4):e38-e47.

101. Morandi A, Piva S, Ely EW, Myatra SN, Salluh JIF, Amare D, et al Worldwide survey of the "assessing pain, both spontaneous awakening and breathing trials, choice of drugs, delirium monitoring/ management, early exercise/mobility, and family empowerment" (ABCDEF) bundle. Crit Care Med 2017;45(11):e1111-e1122.

102. Ely EW, Inouye SK, Bernard GR, Gordon S, Francis J, May L, et al. Delirium in Mechanically Ventilated Patients: Validity and Reliability of the Confusion Assessment Method for the Intensive Care Unit (CAM-ICU). JAMA. 2001;286(21):2703-2710. 